\title{
Aspergillus fumigatus mindert Vitamin-D-Schutz
}

\author{
Auch ohne eine allergische bronchopulmonale Aspergillose \\ (ABPA) findet sich bei Patienten mit zystischer Fibrose (CF) \\ häufig eine Aspergillus fumigatus-Besiedlung. C. A. Coughlan \\ et al. haben den Zusammenhang mit dem Erfolg einer Vita- \\ min-D-Supplementation zur Prävention einer ABPA untersucht. \\ Am J Respir Crit Care Med 2012; 186: 999-1007
}

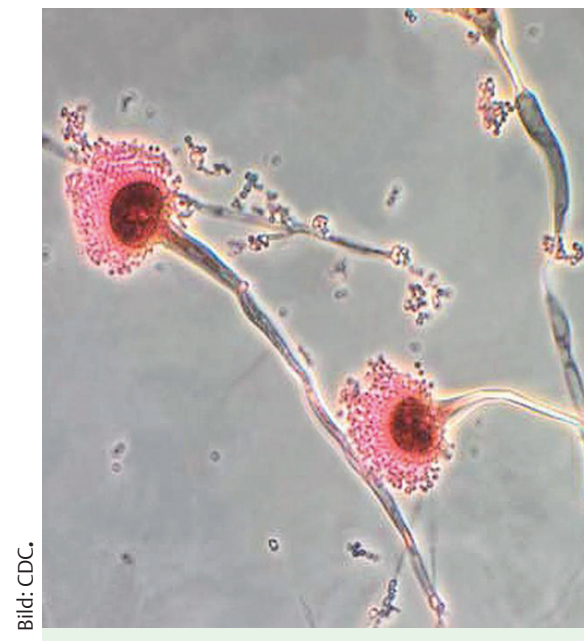

Aspergillus fumigatus ist ein Schimmelpilz aus der Gattung der Gießkannschimmel (Aspergillus).

Ein Vitamin-D-Mangel gilt als ein möglicher Risikofaktor für eine $\mathrm{ABPA}$ bei $\mathrm{CF}$, weil dieser in vitro mit einer erhöhten Interleukin(IL)-5- und IL-13-Produktion einhergeht und die Deletion des VitaminD-Rezeptors ebenfalls eine Erhöhung dieser Zytokine verursacht und zu einer pulmonalen Inflammation führt.

Die vorliegende Studie untersuchte den Zusammenhang von A. fumigatus-Besiedlung ohne ABPA, festgestellt anhand des Sputums und/oder der Flüssigkeit aus einer bronchoalveolären Lavage (BALF) und der nukleären Expression des Vitamin-D-Rezeptors (VDR), die mit Hilfe der quantitativen Real-Time-Polymerase-Kettenreaktion (qRT-PCR) und einem Western-Blot bestimmt wurde. Zusätzlich wurden die IL5- und Il-13-Spiegel per ELISA ermittelt. Den Effekt der Eradikation von A. fumigatus mit Hilfe des Antimykotikums Itraconazol wiesen die Wissenschaftler durch eine Kombination von hochauflösender Computertomografie, Lungenfunktionstestung und Mikrobiologie nach.

\section{Eradikation für Vitamin-D-Effekt essentiel $\nabla$}

Bei einer A.-fumigatus-Besiedlung ohne ABPA war die VDR-Expression in den Epithelzellen der Trachea um $80 \%$ und in den Epithelzellen der Bronchien um 100\% herunterreguliert. Als Ursache identifizierten die Forscher den Pilzmetaboliten Gliotoxin, der in vitro dem positiven Effekt der Gabe von Vitamin D auf die VDRExpression entgegen wirkte und zu einer erhöhten IL-5- und IL-13-Produktion führte. In vivo war die A.-fumigatus-Besiedlung positiv mit einer erhöhten Gliotoxin-Konzentration und erhöhten IL5und IL13-Spiegeln in der BALF assoziiert.

Wurde die A.-fumigatus-Besiedlung der Atemwege mit Hilfe von Itraconazol eradiziert, ließ sich eine Abnahme von Gliotoxin, IL-5 und IL-13 beobachten. Gleichzeitig besserten sich die Atemwegssymptome. Zudem verringerte sich das Mosaikmuster in der hochauflösenden Computertomografie als Hinweis auf eine stabilisierte Lungenfunktion.

\section{Fazit}

Die Ergebnisse weisen auf einen therapeutischen Effekt von Itraconazol bei $\mathrm{CF}$ hin. Gleichzeitig legen sie nahe, dass eine Vitamin-D-Supplementation zur Prävention einer ABPA nur dann erfolgreich ist, wenn $A$. fumigatus eradiziert wird, um die VDR-Expression und damit die positiven funktionellen Effekte des Vitamin D überhaupt zu ermöglichen.

Friederike Klein, München

\section{Atemtest erkennt Lungeninfektionen}

Ein einfacher Atemtest könnte künftig eine rasche Diagnose von Infektionen der Lunge ermöglichen. $\mathrm{Zu}$ diesem Ergebnis kommen Wissenschaftler an der University of Vermont in den USA im Zuge von Experimenten an Mäusen. Die Analyse des Atems sollte die für die Diagnose erforderliche Zeit von Wochen auf Minuten reduzieren.

\section{Organische Verbindungen getestet}

Für die Diagnose bakterieller Infektionen ist es normalerweise erforderlich, eine Probe $\mathrm{zu}$ entnehmen. Die Bakterien werden dann im Labor weitergezüchtet. Für die Klassifikation werden Tests eingesetzt und dadurch ermittelt, welche Reaktion auf Antibiotika erfolgt. Für die aktuelle Studie haben die Forscher flüchtige organische Verbindungen untersucht, die beim Ausatmen von verschiedenen Bakterien, aber auch von verschiedenen Stämmen des gleichen Bakteriums, abgegeben werden.

Mäuse wurden mit 2 Bakterien infiziert, die bei Lungeninfektionen weit verbreitet sind. Dabei handelte es sich um Pseudomonas aeruginosa und Staphylococcus aureus. Atemproben wurden 24 Stunden später gesammelt. Die Bestandteile der Proben wurden mittels SESI-MS analysiert, einer Massenspektrometrie, die extrem kleine Mengen von Chemikalien im Atem erkennen kann. Die Wissenschaftler fanden in der Folge einen deutlichen Unterschied bei den Atemprofilen der infizierten Mäuse und von gesunden Tieren. Zusätzlich konnten die beiden eingesetzten Bakterienarten und 2 Stämme des Bakteriums P. aeruginosa unterschieden werden.

\section{Messverfahren noch unausgereift $\nabla$}

Laut Jane Hill, eine der Autorinnen der Erhebung, sind bei diesem Verfahren noch immer einige Hürden zu überwinden. „Wir arbeiten derzeit mit Kollegen daran, Proben von Patienten zu sammeln, um die Stärken aber auch die Schwäche dieses Ansatzes zu analysieren." Details der Studie wurden Ende 2012 im Fachmagazin ,Journal of Breath Research“ veröffentlicht. 